\title{
The SoLid short baseline neutrino detector
}

\author{
Valentin Pestel ${ }^{* \dagger}$ \\ LPC Caen \\ E-mail: pestel@lpccaen.in2p3.fr
}

The SoLid short baseline reactor neutrino experiment consists in a highly segmented composite scintillator detector with a fiducial mass of 1.6 tons. Its main purpose is to prove or rule out the existence of sterile neutrinos corresponding to $\Delta \mathrm{m}^{2}$ values of order $1 \mathrm{eV}$. The covered baseline ranges between 6 and 9 meters and it is in-line with the compact core of the 60 MW BR2 reactor of the Belgian Nuclear Research Centre. The experiment will come online in the summer of 2017 and will reconstruct more than 50.000 neutrino events per year, based on the inverse beta decay process. In this proceedings we will review the detector technology and several improvements made to the original design, based on the physics and operational performance of a $320 \mathrm{~kg}$ fullsize prototype module that took data at the same site in 2015.

The European Physical Society Conference on High Energy Physics

5-12 July

Venice, Italy

* Speaker.

$\dagger^{\dagger}$ on behalf of the SoLid Collaboration 


\section{Introduction}

The "reactor anti-neutrino anomaly" emerged few years ago, after a recalculation of the flux emitted by nuclear core and an update of the Inverse Beta Decay (IBD) cross section [1]. It consists of a $2.5 \sigma$ deficit in the measured flux by all reactor experiments with baselines shorter than $100 \mathrm{~m}$. When combined with others anomaly (Gallium anomaly and LSND [2]/Mini-BooNe [3] results), it reinforces the hypothesis of the existence of light sterile(s) (i.e. flavourless) neutrino, mixing with the 3 known flavored neutrinos. The "favored" parameters for this oscillation are $\Delta m^{2} \approx 1 \mathrm{eV}^{2}$ and $\sin ^{2}(2 \theta) \approx 0.1$. To probe precisely this $\Delta m^{2}$ value, new measurements are needed at very short baseline $(<10 \mathrm{~m})$. This is the purpose of the SoLid project, located at the BR2 nuclear reactor, at the SCK • CEN in Mol, Belgium. Its experimental program consists on looking for a neutrino flux variation and energy spectrum distortion in accordance with the baseline (6.2 $\mathrm{m}$ to $9.7 \mathrm{~m}$ ). The BR2 core is especially adapted for this measurement with its very compact core $(50 \mathrm{~cm}$ diameter, other parameters are shown on figure 2), reducing the blur on the neutrino emission point. Besides, the very enriched fuel $\left(93.5 \%\right.$ of ${ }^{235} U$ ) simplify the expected flux computation. On the other hand, the SoLid detector have been designed in order to reach a good spatial and energetic resolution. Also, as we operate at a low overburden and near the reactor core, an efficient background rejection is also needed.

\section{SoLid detector overwiew}

Our technology is based on detection cells of $5 \times 5 \times 5 \mathrm{~cm}^{3}$, composed by a plastic scintillator (polyvinyl-toluene or PVT) cube, covered on two faces with thin $(250 \mu \mathrm{m}) \mathrm{LiF}: \mathrm{ZnS}$ layers (see figure 2). The whole elementary cell is optically isolated thanks to a thin Tyvek coating. The light is collected through 4 wavelength shifting optical fibers ( 2 in 2 directions). These fibers are coupled with Silicon-Photo-Multipliers (SiPMs) on one side, and a mirror reflector on the other side. PVT, which is an hydrogen rich material, plays the role of the target for the inverse beta decay reaction : $\bar{v}_{e}+p \rightarrow n+e^{+}$(IBD). The produced positron is directly detected in the PVT and is tagged as the prompt signal. After moderation in the plastic scintillator $(\sim 70 \mu \mathrm{s})$, the neutron can be captured on the ${ }^{6} \mathrm{Li}$ contained in the LiF: $\mathrm{ZnS}$ layer. The breakup reaction $n+{ }^{6} \mathrm{Li} \rightarrow t+\alpha$, releasing $4.77 \mathrm{MeV}$, scintillating in the $\mathrm{ZnS}$. Due to a large difference in the scintillation decay time between PVT ( $\sim 20 \mathrm{~ns})$ and $\mathrm{ZnS}(\sim 10 \mu \mathrm{s})$, a Pulse Shape Discrimination (PSD) is performed to discriminate the two particles. Associated with a time coincidence, this is a powerful signature of the IBD interaction.

These fundamental cells are arranged in planes of $16 \times 16$ cubes, read out by 64 fiber coupled to SiPM. The whole detector is composed of 50 planes, for a global sensible volume of $0.8 \times 0.8 \times$ $2.5 \mathrm{~m}^{3}$. With 8000 voxels $/ \mathrm{m}^{3}$, our detector is able to perform a topological detected events. As IBD signal and background contributions have different topology signature, the segmentation of the fiducial volume allows unique background rejection capabilities.

\section{Efficiency optimisation}

As a shallow depth experiment in proximity of a reactor core, SoLid detector faces a large amount of background : reactor neutrons and gammas, cosmogenic neutrons, cosmic muons. In 

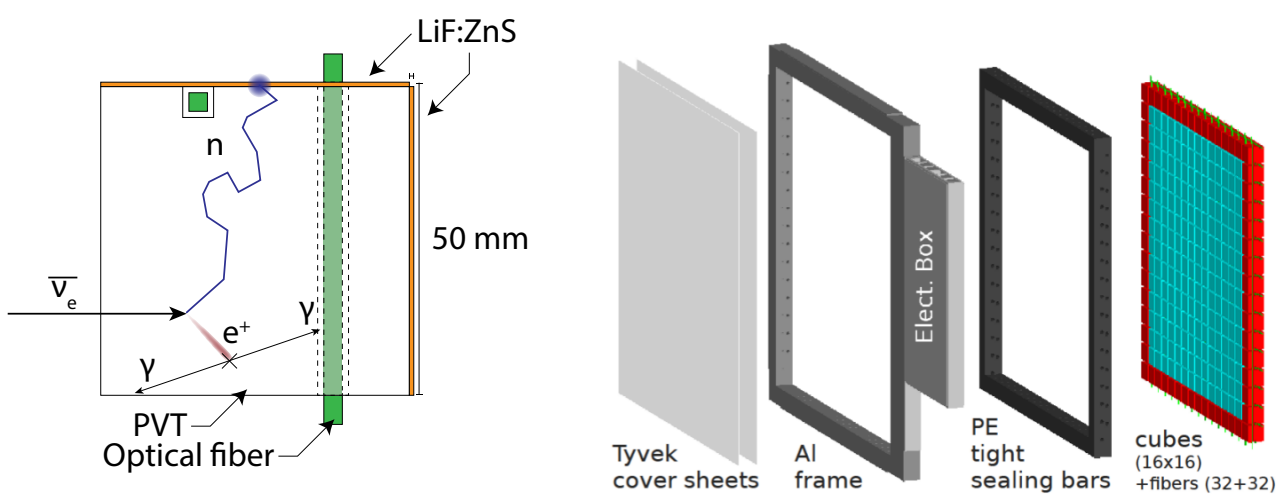

Figure 1: Left : Schematic view of the detection cell. Right : 3D model of a detection plane, with 64 channels (SiPM+fiber+reflector) and 256 detection cells.

\begin{tabular}{r|l} 
SoLid detector \\
\hline Mass & 1.6 Ton \\
IBD detection rate & $300 /$ day \\
IBD efficiency & $30 \%$ \\
Signal/Background & 3 \\
Energy resolution & $\frac{14 \%}{\sqrt{E}}$
\end{tabular}

\begin{tabular}{r|l}
\multicolumn{2}{c}{ BR2 reactor } \\
\hline Thermal power & $50-80 \mathrm{MW}$ \\
Core size & $\varnothing \sim 50 \mathrm{~cm}, h \approx 80 \mathrm{~cm}$ \\
Fuel & $93.5 \%{ }^{235} \mathrm{U}$ \\
Cycles & $\sim 1$ month, 6/years
\end{tabular}

Figure 2: Summary of the SoLid detector and BR2 reactor main characteristics

order to optimize the electronic hardware usage, and reduce needless data flow, the read-out system and especially the trigger must reject as many background as possible. Due to a composite scintillator design, two kinds of signals are expected : a short prompt PVT signal and a delayed long $\mathrm{ZnS}$ signal. The background contribution will be mainly prompt-like signal, corresponding to $\gamma$ interactions in the detector. In this case, a prompt-signal trigger will be ineffective. SoLid approach is to look for the neutron delayed signal as the triggering event, collecting the prompt event in previously buffered signals.

Neutron signals are tagged at the electronic level, using a Peak Over Threshold (POT) algorithm. As the $\mathrm{ZnS}$ light emission is long (more than 256 electronic samples), the waveform is composed of many peaks, each made of few photo-avalanches, as shown on figure 3 . This shape is totally different from the one coming from the PVT emitted light, only grouped one few samples. Instead of looking at a signal above a threshold, we are a looking at the number of peak inside a sliding time window $(6.4 \mu \mathrm{s})$. A comparison of the two approach is shown on figure 3 . In the case of the threshold trigger, the neutron signals are mainly below the hardware limit (black line). The situation is totally different with a POT trigger, allowing a better neutron efficiency. When a neutron event trigger occurs, all the prompt-like signals up to few hundred $\mu s$ before in the neighbour cubes are saved. This part is ensured by a large buffer that store all the signal above a low threshold. This method allows us to reach a high neutron efficiency with a good purity, which is the dominant parameter in the IBD efficiency.

The dark-count rate is an important contribution of the number of peak in a given time window, 

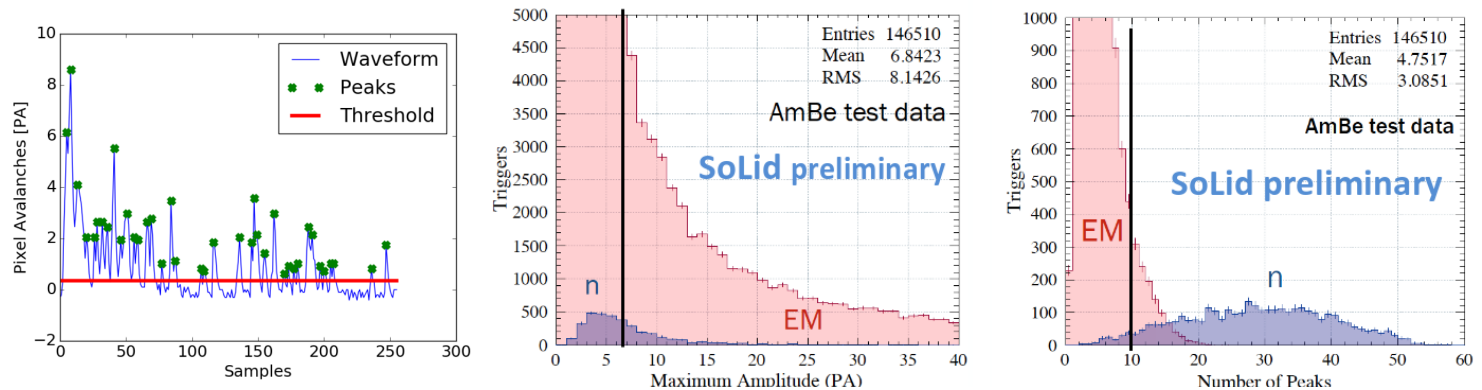

Figure 3: Left : typical neutron event waveform coming from the SoLid read-out. Center and right : respectivelly maximum amplitude and number of peaks distribution for the same AmBe data set. The electron and gamma contributions (EM) are separated from the neutron (n).

increasing the sustainable minimum peak amplitude in a POT trigger scheme. It results in a loss in the neutron efficiency. Taking into account this problem, and in order to avoid this possible issue and increase the neutron detection efficiency, SoLid detector will be operated at $5^{\circ} \mathrm{C}$ thanks to a cooling container. At this temperature, we expect to reduce dark-count rate by a factor of 10 , decreasing the sustainable lower limit in term of number of peak over threshold.

With this trigger system, operated in a cooled environement, SoLid neutron reconstruction efficiency goal is $70 \%$, corresponding to our $30 \%$ IBD efficiency target [5]. All the last improvement discussed here are designed for this purpose [4].

\section{Background shielding}

As very short-baseline experiment operated at sea-level, dominant background is fast-neutron produced by $\mu$ spallation on the high $\mathrm{Z}$ material of building and the core. An efficient shielding should avoid use of this kind of material. Our final background shielding design is based on a water shielding, composed of a $50 \mathrm{~cm}$ thickness water-brick around the detector. For mechanical reasons, the top is made of $50 \mathrm{~cm}$ poly-ethylene plates, as we can see on the whole detector picture (figure 4). According to Monte-Carlo study, validated using the demonstrator [6] data collected during winter 2015, this passive shielding will reduce by at least a factor of 5 the rate of cosmic neutron entering detector, as presented on figure 4.

\section{Construction, Quality assurance and Calibration}

Ensuring homogeneity and calibration at percent level of a detector made of 12800 detection cells and 3200 readout channels is quite challenging. It requires a good tracking of the construction, controlled by a quality assurance step, and a dedicated calibration processes. All components of a detection cell are weighted separately during the construction, as the total cube. Every information is stored in a database with a unique ID. With this last, related to the position of each cube in the detector, the mass repartition of the proton target is accurately known.

After each plane assembly, several measurements are done, using a dedicated robot that moves radioactive source in front of it. These measurements, with ${ }^{22} \mathrm{Na}$ and $\mathrm{Cf}$ source, are designed to check homogeneity in term of light yield and neutron efficiency. It allows us to find and correct 


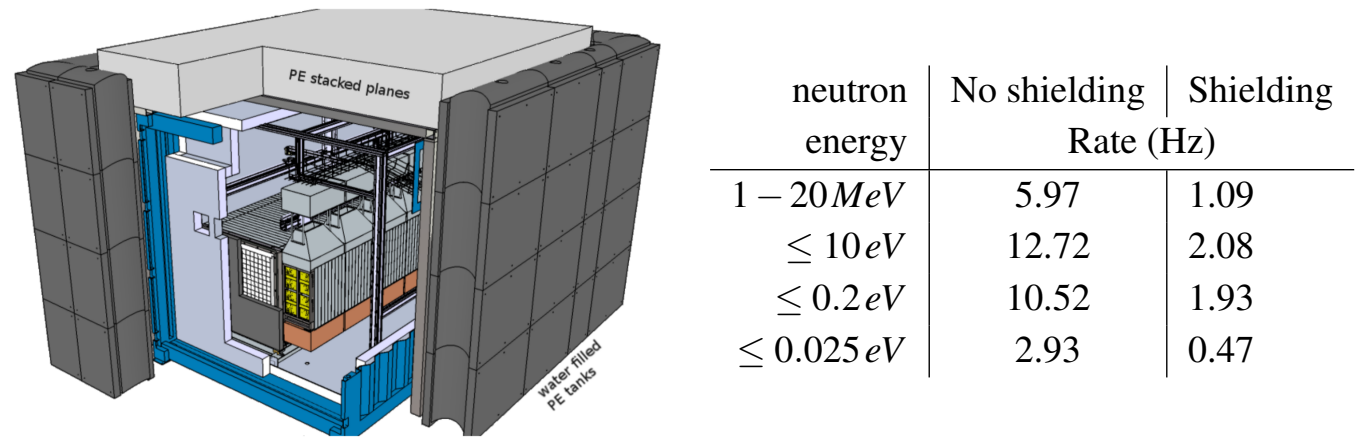

Figure 4: Left : whole view of the final detector design, with an exploded view of a plane and its electronics (in yellow). The cooling system can be seen in orange just below. The container is in blue, surrounded by the water and polyethylene shielding. Right : summary table of the monte-carlo estimation of the rate of cosmic neutrons entering the detector (20 planes configuration).

construction problem when required. This quality assurance step is also an important source of information about our detector, useful to study fine effects.

To reach a percent level measurement, a dedicated process for the $i$ n-situ calibration have been designed. To be efficient and calibrate the large amount of cells with radioactive sources, another dedicated system was designed. This robotic arm can separate module (10 detection planes) and perform a scan with radioactive sources between them, ensuring us a large amount of calibration point. It will be used with two neutron sources (AmBe and $\mathrm{Cf}$ ) previously calibrated at NPL (UK) to know the activity at the percent level. The calibration runs performed with these sources, associated to Monte-Carlo simulation, will determine the neutrons efficiency in the IBD process detection. Radioactive sources $\left({ }^{22} \mathrm{Na},{ }^{137} \mathrm{Cs},{ }^{207} \mathrm{Bi}\right)$ will be used to determine the light-yield in $\mathrm{MeV}$ range, using the same robotic structure. Higher in energy, the gamma coming from AmBe source $(4.4 \mathrm{MeV})$ and neutron capture on hydrogen $(2.2 \mathrm{MeV})$ will be also used. To complete this measurement, the energy loss of the muons $\left(\frac{\Delta E}{d x} \sim 2 \mathrm{MeV} / \mathrm{cm}\right)$ provides information around $10 \mathrm{MeV}$. The energy scale will be well-known and monitored over time all along the interest region $(0-10 \mathrm{MeV})$.

\section{Timeline and conclusion}

SoLid technology relevance and efficiency was already proved by two prototypes [6]. After years of research and development, the construction of the physics scale detector is finishing right now [7]. This new technology required a good monitoring of the construction processes, for what SoLid collaboration has developed a panel of tests. The installation at the BR2 reactor is planned for October this year. As the functionality of the detector plane is checked after the assembly, we expect to complete the commissioning before the end of the year, starting the physics data taking for at least 3 years.

\section{References}

[1] G. Mention et al, The Reactor Antineutrino Anomaly, Phys.Rev.D83,073006 (2011)

[2] A. Aguilar-Arevalo et al. (LSND Collaboration), Phys.Rev.D64,112007 (2001) 
[3] A.A. Aguilar-Arevalo et al. (MiniBooNE), Phys.Rev.Lett.102,101802 (2009) and 105,181801 (2010)

[4] D. NewboldThe SoLid anti-neutrino detector's readout system, proceedings of Topical Workshop on Electronics for Particle Physics (TWEPP2016)

[5] Y. Abreu et al., A novel segmented-scintillator antineutrino detector, JINST 12(04):P04024 (2017)

[6] N. Ryder, First results of the deployment of a SoLid detector module at the SCK •CEN BR2 reactor, PoS(EPS-HEP2015)071

[7] Frederic Yermia, A Search for Sterile Neutrinos with SoLid, these proceedings 